\title{
Mathematical description of super-high frequencies drying process of free-running food media in device with combined energy input
}

\author{
Sergey Antipov ${ }^{1}$, Andrey Klyuchnikov ${ }^{1, *}$, Dmitry Kazartsev ${ }^{2}$ \\ ${ }^{1}$ Voronezh State University of Engineering Technologies, 394036, 19 Revolution Avenue, Voronezh, \\ Russian Federation \\ ${ }^{2}$ Moscow State University of Technologies and Management, 109004, Zemlyanoy Val street, 73, \\ Moscow, Russian Federation
}

\begin{abstract}
At the present time the maim quantity of free-running food products, including grains, are dried in units with convective method of heat input. To intensify convective drying, general attention is paid to improving the method of moving and mixing the product with drying agent, to guarantee fast and quality drying. The use of high and super-high frequency allows can significantly intensify the drying processes, because the phenomenon of super-high frequencies energy into heat conversion throughout the processed material volume contributes to the most uniform heating of the product, compared to other heat input methods. The optimal solution for drying free-running food products is a combination of convective and high-frequency heat input methods. This combination allows controlling gradients of moisture content and temperature, changing its directions, which significantly affects quality of the resulting dry product. In this regard, combined approach to drying process modeling is of practical interest: on the one hand, there are used analytical solutions, based on physical laws application or phenomenological equations, and, on the other hand, experimentally established a relationship between temperature and moisture content of media, which is considered as a heat and mass transfer characteristic for each material.
\end{abstract}

\section{Introduction}

Analysis of existing approaches of drying coriander seeds mathematical description shows, that taking into account only one of the approaches to complex interrelated phenomena modeling occurring during drying (application of the laws of diffusion, thermodynamics or chemical kinetics), it is not possible to describe acceptably all drying periods - at constant and falling speed - with one model. This property can be explained by the fact that the mechanism of moisture movement in the product and the physical Figure of the observed phenomena during drying during these periods, as shown at the Figures $[1-3,7,13]$, are different. Besides, in mathematical description of convective drying with an additional source of super-high frequency energy supply, there emerge

*Corresponding author: kaivanov@mail.ru 
additional difficulties. These difficulties are due to the energy amount mathematical description complexity, which is absorbed by multicomponent substance - coriander seeds.

Drying should be broadly observed as a kind of heterogeneous reaction, defined by a range of chemical, physico-chemical, biochemical and rheological processes, the kinetics of which, along with the kinetics of energy and substance transfer, determine the mechanism and rate of drying processes. Today, in our opinion, there is not enough knowledge has been accumulated about these processes occurring during drying. This fact does not allow us to fully and comprehensively describe it. However, it can be argued that all these drying processes can be described by the equations of chemical kinetics $[5-6,8-12]$.

\section{Objects and research methods}

The methodological basis of the study includes a set of general scientific (analysis and synthesis, verifying the truth of theory by resorting to practice; interpretation of the results, etc.) and private scientific (abstract logical method, modeling, empirical method, statistical probability method, etc.) methods of cognition. The theoretical and methodological basis of research is the work of domestic and foreign authors in the field of drying, in particular, works of Lykov A. V., Rebinder P.A., Ginzburg A.S., Lebedev P. D. etc.

The objects of research were coriander seeds. The hygroscopic properties of coriander seeds were determined by material sorption-desorption isotherms analysis method, obtained experimentally. To identify the intervals of moisture evaporation temperature zones with different shapes and binding energies of moisture with the material, we used the method of differential thermal analysis (DTA) was used. The thermophysical characteristics of edible vegetable raw materials are determined by the method of unsteady thermal regime of $\mathrm{V}$. S. Volkenstein, based on the solution of the problem of thermal conductivity of two temperature-time points. To determine the electrophysical properties of coriander seeds, we used the comparison method (express method), developed at the Plekhanov Russian University of Economics, which allows to determine the dielectric characteristics of a granular product. To find out the range of high-frequency current we used the resonance method of two measurements, consisting in measuring the quality factor and capacitance of the electrical circuit corresponding to the connection of the inductor and the electric capacitor of the Q-meter with empty capacitor and capacitor filled with the product. To determine the content of vitamins, amino acids and other quality indicators of dried raw materials there were used such methods, as high-efficiency gas chromatographyatomic absorption spectroscopy, IR-spectroscopy, capillary electrophoresis, acid hydrolysis etc. The measurement errors did not exceed the values established in the current standards for quantitative analysis methods of dried raw materials quality. The most of theoretical, experimental researches and practical developments carried out in FSBEI HE «Voronezh State University of Engineering Technologies» in accordance with the thematic plans of current research. The goal, formulated in the work, was achieved through generalization and analysis of classical and new analytical and empirical methods for studying heat and mass transfer, based on well-known scientific achievements and fundamental work in the field of food dehydration. The obtained dependences, approximating equations, and simulation results of the studied processes are adequate to experimental data, which is confirmed by measurement results statistical processing. Methodological support and design solutions, proposed as the research result, do not contradict the well-known proven methods of rational design and construction of apparatuses. Set of experiments and implementation of physical and mathematical models of drying and heat-moisture treatment processes were carried out using modern computer mathematical programs, devices and original experimental plant. 


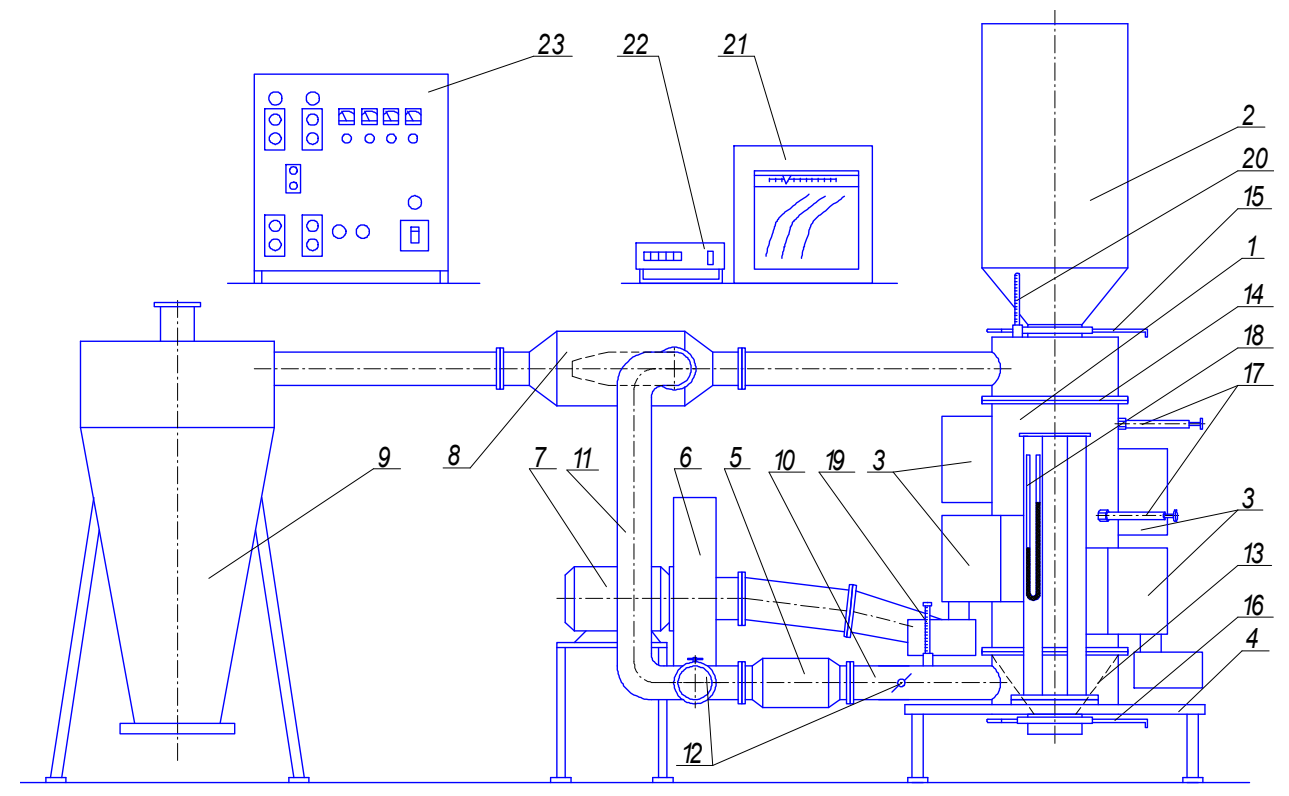

Fig. 1. Scheme of experimental plant.

Description of the experimental plant and experimental methods. Plant (Figure 1) consists of the following main nodes: drying chamber 1 with magnetrons 3 , loading hopper 2 , basement 4 , heating resistance 5 , blower 6 with drive 7 , pipe for air input 10 , exhaust pipe 11, injector 8, cyclone 9 .

The drying chamber is a cylinder by diameter of $0,3 \mathrm{~m}$ and height $0,6 \mathrm{~m}$ coated with thermal insulation. In the middle part of the chamber there are radio-transparent (fluoroplastic) windows through which super-high frequency energy from magnetrons is input 3. Bottom of the drying chamber ends with a perforated cone 13 , and on top with a grid 14. Magnetrons, located on the cylinder, are closed by a casing isolating super-high frequency energy sources from the environment.

Samplers installed along the height of the drying chamber 17 , each allows to select $5 \ldots 10 \mathrm{~g}$ of material from the chamber center without stopping the dryer. The sampler is a hollow cylinder with a small hole on the side surface. Inside the cylinder there is a piston with a slot for selecting material and moving it to the weighing bottle.

\subsection{Development of general structure for the coriander seeds drying process mathematical description}

The central place in the mathematical description of drying is occupied by modeling process speed and material temperature, which make it possible to answer the main questions of engineering calculation - to determine the process duration and the cost of heat to remove moisture.

Comparing different approaches of describing the speed at the first period, it should be noted that the most reliable mathematical model for engineering calculation is a description of the process in the form of a regression equation obtained by processing experimental data:

$$
N_{1}=f\left(x_{1}, x_{2}, \ldots x_{n}\right)
$$


As it is shown by the completed studies analysis $[1-3,4-14,16,17]$, selecting the formula and the required number of regression equation empirical coefficients, it is possible to obtain the formula for determining the speed of the first drying period with the accuracy necessary for engineering calculations.

Material temperature $T$ at moderate drying agent temperatures (less than $373 \mathrm{~K}$ ) should be taken equal to the wet thermometer temperature. With combined energy input, the material temperature will continuously increase due to super-high frequencies heating. Since the temperature increase in the first period is insignificant, then its average value is defined as:

$$
T=T_{M}+a_{0}+a_{1} x_{1}+a_{2} x_{2}+\ldots+a_{n} x_{n},
$$

For the mathematical description of the drying of the second period, the following technique is often used: second period speed $N_{2}(U)$ presented as an expression:

$$
N_{2}(U)=N_{1} \cdot \psi(U),
$$

where $\psi(U)$ - some transfer function of the product moisture content. This function in drying theory is called the relative speed of the second drying period.

The material heating rate in the second drying period is usually estimated by the temperature coefficient or the relative (dimensionless) temperature coefficient of drying.

Relative Drying Temperature Coefficient $B$ is a function of moisture content and does not depend on other factors.

Thus, the drying kinetics mathematical model of the second period will include equation (4.3) and equation of the form:

$$
B=-\frac{\partial T}{\partial U} \cdot \frac{\Delta U}{\Delta T}=f(U),
$$

To obtain an explicit mathematical model of drying kinetics in a period of decreasing speed, it is necessary to carry out additional theoretical and experimental studies.

\section{Development of mathematical description of drying kinetics in decreasing speed period}

For mathematical description of drying kinetics in a period of decreasing speed, we consider drying as a heterogeneous reaction and apply the laws of formal kinetics of chemical reactions to its description:

$$
\frac{\partial \alpha}{\partial \tau}=f(\alpha) \cdot A \cdot \exp \left(-\frac{E}{R T}\right)
$$

where $\alpha=\frac{U_{n}-U}{U_{n}-U_{r}}-$ fractional conversion.

Fractional conversion function can often be represented as:

$$
f(\alpha)=(1-\alpha)^{(n)}=\left(\frac{U-U_{r}}{U_{n}-U_{r}}\right)^{(n)},
$$

For the first period, equation (5) is written as [13]:

$$
-\frac{\partial U}{\partial \tau}=N_{1}=\left(U_{n}-U_{r}\right) \cdot A \cdot \exp \left(-\frac{r}{R T}\right)
$$

In the second period, the bound moisture is removed for which the activation energy is determined by the equation: 


$$
E=r+E_{\mathrm{rm}}=r-R T \ln \phi(U, T),
$$

where $E_{\mathrm{rm}}$ - the moisture binding energy with material, which is determined by the equation of P. A. Rebinder. form:

Then, for the second drying period, the process rate is represented by equation (5) in the

$$
-\frac{\partial U}{\partial \tau}=N_{2}(U)=\left(\frac{U-U_{r}}{U_{\mathrm{cr}}-U_{r}}\right)^{n(U)} \cdot\left(U_{n}-U_{r}\right) \cdot A \cdot \phi(U, T) \cdot \exp \left(-\frac{E}{R T}\right)
$$

To describe fully the kinetics of drying in a period of decreasing speed, we supplement equation (9) with the equation of the temperature curve (4).

For colloidal capillary-porous bodies, the coefficient B can be represented as:

$$
B=-\frac{\partial T}{\partial U} \cdot \frac{\Delta U}{\Delta T}=a \cdot \exp \left[-m\left(U-U_{r}\right)\right],
$$

We will accept the fixed values of temperature and moisture content:

$$
\Delta U=U_{\mathrm{cr}}-U_{r} ; \Delta T=T_{k}-T_{\mathrm{cr}} ;
$$

where $T_{\mathrm{cr}}$ - material temperature upon reaching critical moisture content, determined by the equation (2); $T_{\mathrm{k}}$ - material temperature when reaching equilibrium moisture content, presented in the form:

$$
T_{k}=f\left(T_{\mathrm{s} . \mathrm{a}}, P_{\mathrm{st}}\right) .
$$

Coefficient $m$ does not depend on the drying mode and is a constant value for a given material.

Equation (10) in many cases satisfactorily describes the experimental data at the range $U_{\text {cr }}<U<U_{\mathrm{r}}$. However, the application of equation (10) at $U=U_{\mathrm{cr}}$ leads to a violation of the relationship between $N_{2}(\mathrm{U})$ and $N_{1}$, since according to (10) the value $\frac{\partial T\left(U_{\mathrm{cr}}\right)}{\partial U} \neq 0$. Therefore, we represent the dependence of the relative temperature coefficient of drying by the equation:

$$
B=-\frac{\partial T}{\partial U} \cdot \frac{\Delta U}{\Delta T}=a \cdot\left\{\exp \left[m\left(U_{\mathrm{cr}}-U\right)\right]-1\right\} .
$$

We find the material temperature by integrating the equation (12):

$$
T=a \frac{\Delta T}{\Delta U} \cdot\left\{\frac{1}{m} \exp \left[m\left(U_{\mathrm{cr}}-U\right)\right]+U\right\}+C .
$$

We find the integration constant from the condition: by $U=U_{\mathrm{cr}} ; T=T_{\mathrm{cr}}$. Under this condition, we obtain:

$$
C=T_{\mathrm{cr}}-a \frac{\Delta T}{\Delta U} \cdot\left\{\frac{1}{m} \exp \left[m\left(U_{\mathrm{cr}}-U\right)\right]+U_{\mathrm{cr}}\right\}
$$

The integration constant can also be determined from the condition: by $U=U_{\mathrm{p}}$; $T=T_{\mathrm{\kappa}}$.

$$
C=T_{\mathrm{cr}}-a \frac{\Delta T}{\Delta U} \cdot\left(\frac{1}{m}+U_{\mathrm{cr}}\right) .
$$

Equating the right-hand sides of equations (14) and (15), we obtain: 


$$
T_{\mathrm{cr}}-a \frac{\Delta T}{\Delta U} \cdot\left\{\frac{1}{m} \exp \left[m\left(U_{\mathrm{cr}}-U\right)\right]+U_{\mathrm{cr}}\right\}=T_{\mathrm{cr}}-a \frac{\Delta T}{\Delta U} \cdot\left(\frac{1}{m}+U_{\mathrm{cr}}\right)
$$

Thence:

$$
a=\frac{\Delta U}{\frac{1}{m}[\exp (m \cdot \Delta U)-1]-\Delta U} .
$$

Material temperature taking into account (15) and (17) is equal to:

$$
T=T_{\mathrm{cr}}+\left(T_{k}-T_{\mathrm{cr}}\right) \cdot \frac{\frac{1}{m}\left\{\exp \left[m\left(U_{\mathrm{cr}}-U\right)\right]-1\right\}-\left(U_{\mathrm{cr}}-U\right)}{\frac{1}{m}\left\{\exp \left[m\left(U_{\mathrm{cr}}-U_{r}\right)\right]-1\right\}-\left(U_{\mathrm{cr}}-U_{r}\right)} .
$$

Of practical interest is the temperature curve equation written in generalized variables:

$$
\frac{T-T_{\mathrm{cr}}}{T_{k}-T_{\mathrm{cr}}}=\frac{\frac{1}{m}\left\{\exp \left[m\left(U_{\mathrm{cr}}-U\right)\right]-1\right\}-\left(U_{\mathrm{cr}}-U\right)}{\frac{1}{m}\left\{\exp \left[m\left(U_{\mathrm{cr}}-U_{r}\right)\right]-1\right\}-\left(U_{\mathrm{crp}}-U_{r}\right)} .
$$

By analogy with [13], we denote:

$\alpha_{T}=\frac{T-T_{\text {cr }}}{T_{k}-T_{\text {cr }}}$ - heating degree of the dry matter in the second drying period, which is determined by the ratio of heat, spent on heating the dry part of the material at a time $\tau$, to the heat, spent on heating this substance for the entire second drying period; $\alpha_{U}=\frac{\left(U_{\mathrm{cr}}-U\right)}{\left(U_{\mathrm{cr}}-U_{r}\right)}-$ substance drying degree; $U_{\mathrm{cr}}-U=\alpha_{U} \cdot \Delta U$,

then equation (19) takes form:

$$
\alpha_{T}=\frac{\frac{1}{m}\left\{\exp \left[m\left(U_{\mathrm{cr}}-U\right)\right]-1\right\}-\alpha_{U} \cdot \Delta U}{\frac{1}{m}\left\{\exp \left[m\left(U_{\mathrm{cr}}-U_{r}\right)\right]-1\right\}-\Delta U} .
$$

Taking into account equations (12) and (17), the relative temperature coefficient of drying will have form:

$$
B=\frac{\Delta U\left\{\exp \left[m\left(U_{\mathrm{cr}}-U\right)\right]-1\right\}}{\frac{1}{m}\{\exp [m \cdot \Delta U]-1\}} .
$$

Find the drying speed for the second period.

Transform the equation exponent (9):

$$
\exp \left(-\frac{E}{R T}\right)=\phi(U, T) \cdot \exp \left(-\frac{r}{R T_{\mathrm{cr}}}\right) \cdot \exp \left(\frac{r}{R T_{\mathrm{cr}}} \cdot \frac{T-T_{\mathrm{cr}}}{T}\right) .
$$

Then we write the equation of the drying rate of the second period in the form:

$$
N_{2}(U)=N_{1}\left(\frac{U-U_{r}}{U_{\mathrm{cr}}-U_{r}}\right)^{n(U)} \cdot \phi(U, T) \cdot \exp \left(\frac{r}{R T_{\mathrm{cr}}} \cdot \frac{T-T_{\mathrm{cr}}}{T}\right) .
$$

We denote:

$$
Z(U)=\left(T_{k}-T_{\mathrm{cr}}\right) \cdot \frac{\frac{1}{m}\left\{\exp \left[m\left(U_{\mathrm{cr}}-U\right)\right]-1\right\}-\left(U_{\mathrm{cr}}-U\right)}{\frac{1}{m}\left\{\exp \left[m\left(U_{\mathrm{cr}}-U_{p}\right)\right]-1\right\}-\left(U_{\mathrm{cr}}-U_{r}\right)} .
$$

Then the expression for the drying rate of the second period has the form:

$$
N_{2}(U)=N_{1}\left(\frac{U-U_{r}}{U_{\mathrm{cr}}-U_{r}}\right)^{n(U)} \cdot \phi(U, T) \cdot \exp \left(\frac{r}{R T_{\mathrm{cr}}} \cdot \frac{Z(U)}{Z(U)+T_{\mathrm{cr}}}\right) .
$$


The comparative speed super-high frequencies drying of the second period for coriander seeds is:

$$
\psi(U)=\frac{N_{2}}{N_{1}}=\left(\frac{U-U_{r}}{U_{\mathrm{cr}}-U_{r}}\right)^{n(U)} \cdot \phi(U, T) \cdot \exp \left(\frac{r}{R T_{\mathrm{cr}}} \cdot \frac{Z(U)}{Z(U)+T_{\mathrm{cr}}}\right)
$$

\section{Calculation of the drying process duration based on equivalent moisture content}

The resulting mathematical model allows to determine the drying process duration:

$$
\begin{gathered}
\tau=\tau_{1}+\tau_{2}, \\
\tau_{1}=\frac{U_{n}-U_{\mathrm{cr}}}{N_{1}}, \\
\tau_{2}=\int_{U_{k}}^{U_{\mathrm{cr}}} \frac{d U}{N_{2}(U)}=\frac{1}{N_{1}} \int_{U_{k}}^{U_{\mathrm{cr}}} \frac{d U \cdot \exp \left[-\frac{r}{R T_{\mathrm{cr}}} \cdot \frac{Z(U)}{Z(U)+T_{\mathrm{cr}}}\right]}{\left(\frac{U-U_{r}}{U_{\mathrm{cr}}-U_{r}}\right)^{n} \cdot \phi(U, T)} .
\end{gathered}
$$

Having written explicitly the equation of the desorption curve $\varphi(U, T)$ the integral can be calculated using numerical methods. However, we can use another calculation method, based on the determination of equivalent moisture content.

We denote that when drying the product from $U_{\mathrm{n}}$ to $U_{\mathrm{k}}$ equality is respected:

$$
N_{1} \cdot \tau=N_{1} \cdot \tau_{1}+N_{1} \cdot \tau_{2}=W_{1}+W_{2}=W_{\text {ch }} \approx \text { const. }
$$

This equality is possible if we take the quantities independent of the drying regimes $U_{\text {cr }}$ and $\varphi(U, T)$, which can be admited in the temperature range under study, with a certain degree of accuracy. We call the value of $W$ the equivalent moisture content.

To calculate the equivalent moisture content, the basic equation of drying kinetics is proposed in the following form:

$$
d W=\frac{q_{1}}{q_{2}(U)} \cdot d U \cdot\left[1+\frac{E_{\mathrm{rm}}}{r}+\frac{c_{c}+U \cdot c_{B}}{r} \cdot \frac{d T}{d U}\right],
$$

In our case, as was previously shown, the analytical determination of these heat flows is difficult. Therefore, to approximate the function $W=N_{1} \cdot \tau=f\left(U_{n}, U_{k}\right)$ the experimental research is needed. Next, it is necessary to determine $W$ for various values of Un, Uk and choose an empirical equation to describe it.

Using dependencies $W=f\left(U_{n}, U_{k}\right)$ and $N_{1}=f\left(x_{1}, x_{2}, \ldots x_{n}\right)$ it is possible to calculate the drying time under any conditions in the set interval of the change of process factors.

\subsection{Experimental verification of the equation of heat and mass transfer association}

For the period of the decreasing drying rate, we have established in explicit form the relationship between heat and mass transfer in the form of the equation (31). For experimental verification of the obtained equation, the experimental data on the coriander seeds drying was processed in the form of graphical dependence $\alpha_{T}=f\left(\alpha_{U}\right)$, Figure 2. The same graph shows the experimental data of derivatographic study of drying coriander and a theoretical curve of heat and mass transfer relationship, calculated according to equation (31) for $m=17$. The coefficient $m$ was determined from experimental data using the least squares method. 
The relationship (31) is not affected by either the design of the apparatus or the temperature drying regime. It should be noted that in our case, the coefficient $m$ is influenced by the initial moisture content of coriander seeds, since the ratio of the amount of free and bound moisture changes with initial moisture content changing.

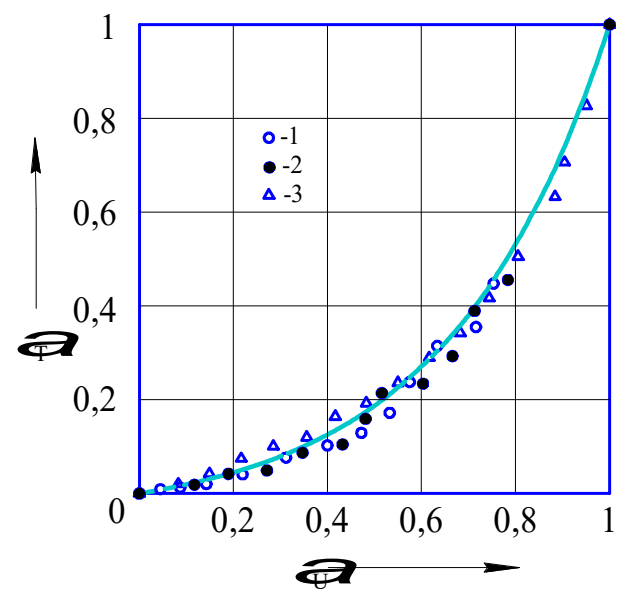

Fig. 2. Drying coriander seeds in experimental plant within $U_{\mathrm{H}}=0,18 \mathrm{~kg}_{\mathrm{m}} / \mathrm{kg}$, d.v: $1-T_{\mathrm{sa}}=348 \mathrm{~K}$, $Q_{\mathrm{sa}}=197,82 \mathrm{~m} 3 / \mathrm{h}, P_{\mathrm{st}}=240 \mathrm{~W} / \mathrm{kg}_{\mathrm{d} . \mathrm{v}} ; 2-T_{\mathrm{sa}}=318 \mathrm{~K}, Q_{\mathrm{ca}}=197,82 \mathrm{~m}^{3} / \mathrm{h}, P_{\mathrm{st}}=240 \mathrm{~W} / \mathrm{kg}_{\mathrm{d} . \mathrm{v} \cdot} \cdot 3-$ drying in derivatograph oven $U_{\mathrm{H}}=0,176 \mathrm{~kg}_{\mathrm{m}} / \mathrm{kg}_{\mathrm{s} . \mathrm{v}}, d T / d \tau=1,25 \mathrm{~K} / \mathrm{min}$.

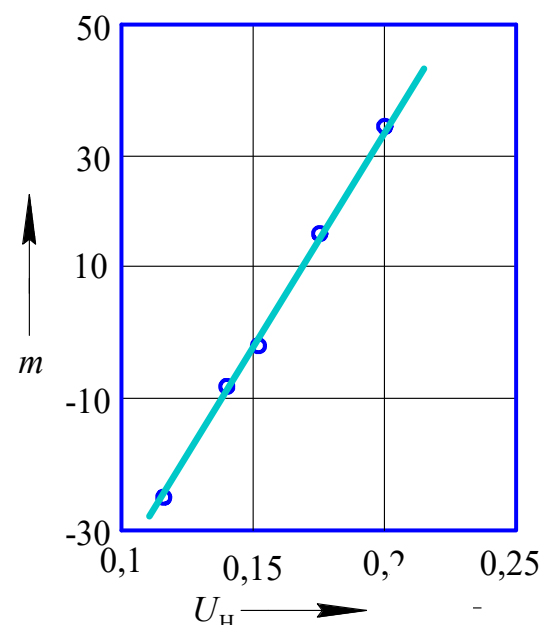

Fig. 3. Dependence of the kinetic coefficient $m$ on the initial moisture content of coriander seeds.

For experimental test of the scientific hypothesis about the possibility of applying the kinetics laws of chemical reactions in mathematical modeling of convective-high-frequency drying and finding empirical coefficients included in mathematical model equations, we studied the drying of coriander seeds in a unit with a combined energy supply.

As it is shown at the Figure 3, the dependence of the coefficient $m$ on the initial moisture content is linear. An empirical equation was chosen to approximate this dependence:

$$
m=660,49 \cdot U_{H}-101,91 .
$$


It is important that the relationship (20) is not affected by the supplied super-high frequencies power. Therefore, kinetic coefficient $m$ determination is possible according to the laboratory analysis results.

\section{Matching of theoretical and experimental drying temperature curve}

As it was mentioned earlier, the material temperature is a function of moisture content, temperature of the drying agent and super-high frequencies power and is practically independent of other factors.

Figure 4 presents theoretical calculations of the coriander seeds temperature in a period of decreasing speed using the equations (18), (20) at $m=4$. For comparison, the experimental data obtained at various temperatures of the drying agent and super-high frequencies power are plotted.

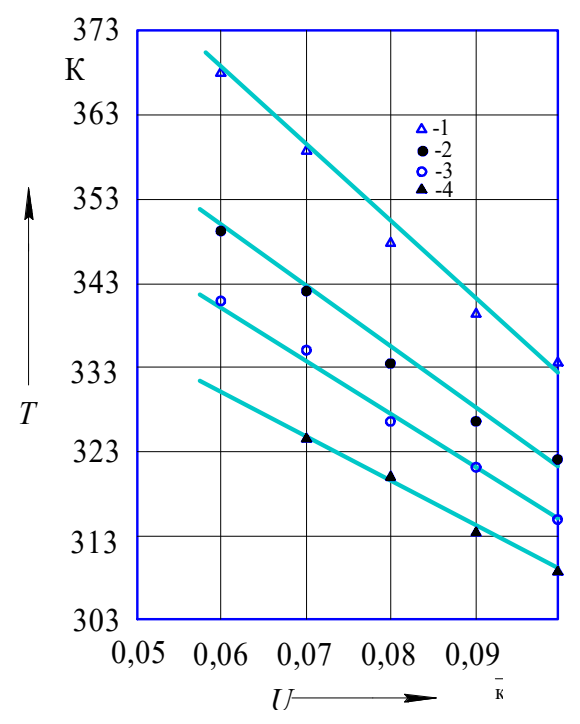

Fig. 4. Changing of coriander seeds temperature in decreasing drying speed period (theoretical calculation). Experimental data within $U_{\mathrm{n}}=0,16 \mathrm{~kg}_{\mathrm{m}} / \mathrm{kg}_{\mathrm{d} . \mathrm{y}}: 1-T_{\mathrm{sa}}=363 \mathrm{~K}, Q_{\mathrm{sa}}=141,3 \mathrm{~m}^{3} / \mathrm{h}, P_{\mathrm{st}}=$ $180 \mathrm{~W} / \mathrm{kg} ; 2-T_{\mathrm{sa}}=333 \mathrm{~K}, Q_{\mathrm{sa}}=141,3 \mathrm{~m}^{3} / \mathrm{h}, P_{\mathrm{st}}=300 \mathrm{~W} / \mathrm{kg} ; 3-T_{\mathrm{sa}}=333 \mathrm{~K}, Q_{\mathrm{sa}}=141,3 \mathrm{~m}^{3} / \mathrm{h}, P_{\mathrm{st}}=$ $180 \mathrm{~W} / \mathrm{kg} ; 4-T_{\mathrm{sa}}=333 \mathrm{~K}, Q_{\mathrm{sa}}=141,3 \mathrm{~m}^{3} / \mathrm{h}, P_{\mathrm{st}}=60 \mathrm{~W} / \mathrm{kg}$.

As can be seen, the theoretically examined dependence of coriander seeds temperature on the moisture content in the decreasing drying period rate corresponds to this dependence, constructed from experimental data.

\section{Results and discussion}

When choosing drying coriander seeds method, along with energy consumption for the process and drying time, it is necessary to take into account the end product quality, which largely depends on the color of seeds and the content of essential oils inside. Therefore, it is necessary, that during the drying process these parameters are retained, and if it is possible should be improved.

It was found that super-high frequencies heating of coriander seeds for $\begin{array}{lllll}0.5 & \ldots & 2.0\end{array}$ minutes allows to increase the yield of essential oil by $13 \%$ from the original. 
To establish the influence of super-high frequencies energy on the coriander seeds quality indicators, the organoleptic and physico-chemical parameters of the dried product were determined with convective and combined energy input. The drying process parameters were selected on the basis of the experiments and amounted to:

- with convective drying: $U_{\mathrm{n}}=0,16 \mathrm{~kg}_{\mathrm{m}} / \mathrm{kg}_{\mathrm{d} . \mathrm{v}}, T_{\mathrm{sa}}=373 \mathrm{~K}, Q_{\mathrm{sa}}=150 \mathrm{~m} / \mathrm{h}$;

- when drying with super-high frequencies energy input: $U_{\mathrm{n}}=0,16 \mathrm{~kg}_{\mathrm{m}} / \mathrm{kg}_{\mathrm{d} . \mathrm{v}}, T_{\mathrm{sa}}=373$ $\mathrm{K}, Q_{\mathrm{sa}}=141,3 \mathrm{~m}^{3} / \mathrm{h}, P_{\mathrm{st}}=200 \mathrm{~W} / \mathrm{kg}$.

It should be noted that it is undesirable to use constant drying modes for periods of constant and decreasing speed, because the use of preferred modes for one drying period in most cases can adversely affect the quality of the resulting product and energy consumption with another drying period.

When drying coriander seeds with a high initial moisture content of $0,16 \mathrm{~kg}_{\mathrm{m}} / \mathrm{kg}_{\mathrm{d} . \mathrm{v}}$ and more in the first drying period, it is desirable to use low temperature drying agent $-323 \ldots 333 \mathrm{~K}$, and the lower the temperature, the higher the initial moisture content. However, the use of drying agent with temperatures below $313 \mathrm{~K}$ leads to the fact that the heat generated in the product under the influence of super-high frequencies energy is spent not only on the evaporation of moisture, but also on the drying agent heating, which negatively affects energy consumption. The use of current temperatures provides more uniform thermal effect on the product and greater drying uniformity along the height of the layer. When drying coriander seeds with a low initial moisture content, the temperature of the drying agent in the first drying period can be slightly increased.

The supplied super-high frequencies power in the first drying period must not exceed $180 \ldots 200 \mathrm{~W} / \mathrm{kg}_{\text {d. v. }}$, otherwise it can lead to a large gradient of internal pressure, volumetric stress state inside the material, cracks formation and destruction of the coriander seeds structure and, ultimately, significant loss of essential oils. Increasing super-high frequencies power to $300 \mathrm{~W} / \mathrm{kg}_{\mathrm{s} \text { d. v. }}$ may also cause product burning. It should be noted that an increase in the supplied super-high frequencies power by 1.2 times leads to a reduction in the drying time by 1.5 times, but leads to an increase in energy consumption.

It was noted that drying agent consumption increase intensifies the process only in the first drying period and practically does not affect the rate in the second drying period. This implies, that in the first period it is desirable to use high speeds of the drying agent, of the order of $1 \mathrm{~m} / \mathrm{s}$ and higher, depending on the drying unit design, to reduce it as the moisture content decreases, in order to reduce the material temperature increasing intensity in the second period and the energy consumption for the process $[4,15]$.

\section{Conclusions}

The proposed mathematical description of the super-high frequencies drying process of free-running food media in device with combined energy input has a number of positive aspects: firstly, it is considered separately for a period of constant drying rate using an empirical model and for a period of decreasing speed, based on the applied laws of chemical kinetics; secondly, it allows to answer the main questions due carrying out engineering calculations of drying equipment, namely, determining the duration of the drying process and the cost of heat to remove moisture; thirdly, to formulate requirements for raw materials quality, drying process parameters, from the point of view of high efficiency of the technology organization of its processing with combined energy input.

Thus, the use of combined energy input can significantly accelerate the drying process and improve the organoleptic and physico-chemical characteristics of raw material.

\section{References}


1. J. Ahmed, U.S. Shivhare, G. Singh, Food and Bioproducts Processing: Transactions of the Institution of Chemical Engineers, Part C 79(2), 103 - 106 (2001) DOI: $10.1205 / 096030801750286258$

2. J.R. Arballo, L.A. Campañone, R.H. Mascheroni, Drying Technology 28(10), 1178 1184 (2010) DOI: 10.1080/07373937.2010.493253

3. I. Boshkova, N. Volgusheva, A. Titlov, S. Titar, L. Boshkov, Eastern-European Journal of Enterprise Technologies 1(8-97), 78 - 85 (2019) DOI: 10.15587/17294061.2019.154527

4. P. Divya, B. Puthusseri, B. Neelwarne, Food Research International 45(1), $342-350$ (2012) DOI: 10.1016/j.foodres.2011.09.021

5. A. Ghasemi Pirbalouti, S. Salehi, L. Craker, Journal of Applied Research on Medicinal and Aromatic Plants 4, 35 - 40 (2017) DOI: 10.1016/j.jarmap.2016.07.006

6. Q. Guo, D.-W. Sun, J.-H. Cheng, Z. Han, Trends in Food Science and Technology 67, 236 - 247 (2017) DOI: 10.1016/j.tifs.2017.07.007

7. M. Hemis, R. Choudhary, N. Becerra-Mora, P. Kohli, V. Raghavan, International Journal of Agricultural and Biological 9(6), 167 - 177 (2016) DOI: 10.3965/j.ijabe.20160906.2442

8. S. Hihat, H. Remini, K. Madani, International Food Research Journal 24(2), 503 - 509 (2017) ISSN: 19854668

9. B. Kozanoglu, Á. Sánchez-Huerta, J.A. Guerrero-Beltrán, J. Welti-Chanes, Chemical Engineering Communications 203(9), 1227 - 1233 (2016) DOI: 10.1080/00986445.2016.1160377

10. V.I. Markin, M.Yu. Cheprasova, N.G. Bazarnova, Russian Journal of Bioorganic Chemistry 41(7), 686 - 699 (2015) DOI: 10.1134/S1068162015070110

11. I.K. Masalimov, S.F. Faizrakhmanov, I.I. Gabitov, A.A. Davydova, I.R. Rakipov, A.S. Ramazanov, Journal of Engineering and Applied Sciences 13, 6570 - 6575 (2018) DOI: 10.3923/jeasci.2018.6570.6575.

12. S.K. Mustafayev, T.V. Pelipenko, A.P. Usov, E.A. Kalienko, International Journal of Pharmacy and Technology 8(4), $27334-27350$ (2016) ISSN: 0975766X

13. T. Sadi, S. Meziane, International Food Research Journal 22(2), 494 - 501 (2015) ISSN: 19854668

14. A. Sarimeseli, Energy Conversion and Management 52(2), 1449 -1453 (2011) DOI: 10.1016/j.enconman.2010.10.007

15. M. Shaw, V. Meda, L. Tabil Jr., A. Opoku, Journal of Microwave Power and Electromagnetic Energy 41(2), 59 - 68 (2007) ISSN: 08327823

16. N. Volgusheva, E. Altman, I. Boshkova, A. Titlov, L. Boshkov, Eastern-European Journal of Enterprise Technologies 6(8-90), 47 - 54 (2017) DOI: 10.15587/17294061.2017.115118

17. Y.-Y. Xu, S.-S. Cai, H.-C. Wu, Jilin Daxue Xuebao (Gongxueban)-Journal of Jilin University (Engineering and Technology Edition) 44(2), 579 - 584 (2014) DOI: 10.13229/j.cnki.jdxbgxb201402047

18. D. Zhu, J. Wang, D. Zhu, P. Xia, J. Zhou, N. Zhang, Nongye Gongcheng XuebaoTransactions of the Chinese Society of Agricultural Engineering 23(12), 242 - 246 (2007) ISSN: 10026819 